\title{
ISAR Image Classification with Wavelet and Watershed Transforms
}

\author{
B. Mamatha ${ }^{1}$, V. Valli Kumar ${ }^{2}$ \\ ${ }^{1}$ NSTL, Visakhapatnam, India \\ ${ }^{2}$ Department of Computer Science \& System Engineering, Andhra University, Visakhapatnam, India
}

\begin{tabular}{|c|c|}
\hline Article Info & ABSTRACT \\
\hline Article history: & Inverse Synthetic Aperture Radar images are playing a significant role in \\
\hline Received Jul 8, 2016 & $\begin{array}{l}\text { classification of sea and air targets. First we acquire the ISAR images of } \\
\text { targets using a sensor like radar and extract the characteristics of targets from }\end{array}$ \\
\hline Revised Sep 9, 2016 & the ISAR images in the form of feature vectors. The computed feature \\
\hline Accepted Sep 23, 2016 & vectors are used for classification of targets. In this work, widely used and \\
\hline Keyword: & $\begin{array}{l}\text { technique wavelet transform are explored to derive the target features. An } \\
\text { artificial neural network based classifier is used for classification. The }\end{array}$ \\
\hline Classification accuracy & Wavelet analysis divides the information of an image into approximation and \\
\hline Classifier & detail sub signals. The approximate and three detail sub signal values are \\
\hline Color moments & taken as feature vectors and given as input to the classifier for ship ISAR \\
\hline Isar image & image classification. The widely used segmentation technique, Watershed \\
\hline Watershed transform & transform is applied to the ISAR images. The wavelet coefficients are \\
\hline Wavelet transform & $\begin{array}{l}\text { computed for the segmented ISAR images and used as feature vectors for } \\
\text { classification of the ISAR images. Also, the statistical moments mean and } \\
\text { standard deviation are computed for the color ISAR images itself, taken in } \\
\text { RGB format. These statistical color moments are used as feature vector. The } \\
\text { classification accuracy is compared for the feature vectors. }\end{array}$ \\
\hline
\end{tabular}

Copyright (c) 2016 Institute of Advanced Engineering and Science. All rights reserved.

\section{Corresponding Author:}

B. Mamatha,

Naval Science \& Technological Laboratory, Vigyannagar, Visakhapatnam-530027, India.

Email: mamathabasava67@gmail.cm

\section{INTRODUCTION}

Classification of targets has immense importance in defense and civilian applications like coastal surveillance, prevention of unauthorized fishing etc. Classification is to assign an unknown target to a class from a given set of classes. The classification is based on attribute values of the targets obtained from different representations of targets. In this work Inverse Synthetic Aperture Radar images generally measured with a high resolution Radar are used for target feature extraction. The ISAR images represent the two dimensional distribution of target scatterers in down range (line of site of radar) and cross range (perpendicular to down range) directions. The measurement of ISAR images is a very complex task that requires uniform motion of the target with respect to radar. The formation of an ISAR image involves highly computational signal processing. The role, pitch and yaw information of the targets in case of ships is also considered along with the target echo data to synthesize an ISAR image Neural networks are widely being used in various intelligent systems. One such computer vision system for recognition and classification of commercial cork tiles is described in [1]. Various feature vector extraction methods based on color, texture and shape can be seen in [2]. The comparison of classification performance of different feature vectors average RGB, color moments, color histograms etc. is given in [3]. How the feature representation and feature extraction are rqually critical in multimedia processing is explained in [4]. In this work a probabilistic neural network is used for ISAR image classification. The classifier performance accuracy presented in this 
paper is done with ISAR images of two ships that are measured with stepped frequency waveform radar. For feature extraction, an ISAR image is considered as a digital image represented with a two dimensional matrix of pixel intensity values. In this study mathematical transforms Watershed transform and wavelet transform are applied to the ISAR images to extract target attributes.

The watershed transform is a mathematical transform that can be categorized as a region based image segmentation technique. The segmentation refers to subdividing an image into its constituent regions. In segmentation the image elements that exhibit similar characteristics are grouped. The segmentation results obtained from watershed transform can be taken as input for further image analysis. Segmentation is a critical image processing task for accurate interpretation of certain images like medical images used for medical diagnosis. Application of Watershed segmentation to medical images gives essential information useful for better medical diagnosis [5]. An object oriented approach for unsupervised classification of high resolution remotesensing images is proposed. The fractal net evaluation technique is used as an image segmentation technique for extraction of objects from the images [6]. The usefulness and effectiveness of using Watershed segmentation in detection of oral cancer and tumors in mammograms is explained in [7-8]. In our work, Watershed segmentation is applied to the ship ISAR images before feature extraction.

Wavelet Transform is a multi resolution technique often used to resolve some parts of signal in time and some other parts in frequency. The wavelet coefficients, the approximation, horizontal, vertical and detail coefficients are widely being used as feature vectors in various image classification and image retrieval applications. The statistical moments of wavelet detail coefficients are used for ECG image classification [9]. The study and analysis of electromyography signals have application in sports science. These electro-physiological signals are used to determine muscle strength, muscle force etc. that determine the human ailments like low back pain etc. The wavelet transform is used for the feature extraction and classification of EMG signals [10]. Heart Rate Variability (HRV) signals represent a human's Autonomous Nervous System. Wavelet coefficients computed for the HRV's are used as feature vectors to represent the signals and their further analysis [11]. In gender classification through face images, the logarithmic wavelet of Gabor is used to separate data related to texture from internmediate frequency bands and use the data in image segmentation. The local binary patterns of the segmented images are used for gender classification [12]. The wavelet transform is also used for image compression. Images contain large amount of information that requires much storage space. Many a time it is very useful to compress the image. Images contain redundant information. This redundancy feature is exploited to compress images. Only the essential information needed to reconstruct an image is only stored. Wavelets are also used for segmentation of images.

The computational overhead of conversion of color image to gray image before feature extraction can be avoided by extraction of feature vectors from the color ISAR images and used for classification. The color images are represented in different color models like RGB, Indexed image, NTSC, YCbCr, CMY, CMYK and HSV. The image in one model can be converted to any other model by suitable transformation. Each of these models is suitable for one particular application [13]. In this work RGB color model is taken and successfully used for feature extraction and ship ISAR image classification. Color feature is an efficient visual characteristic of images and so the color images are being used extensively in content based image retrieval from huge databases. The single color moments and two color moments are being used for ISAR image classification [14]. RGB color histogram and Canny edge detection is used as a feature vector for image retrieval from a database [15]. An important characteristic of color feature is invariance to rotation which is very essential for ISAR image classification since ISAR images are aspect variant.

\section{FEATURE EXTRACTION}

\subsection{Wavelet Transform}

The continuous wavelet transform is the sum over time of scaled and shifted wavelet $\Psi$ and is given

$$
C(s, t)=\int_{-\infty}^{\infty} f(t) \Psi(s, t, \tau) d t
$$

Where's' is scale of a wavelet which is proportional to the inverse of the frequency information. The translation $\tau$ is proportional to time information. Wavelets are mathematical functions that divide data into different frequency components. The image can be decomposed using low pass and high pass filter. This low pass and high pass filter pair is called analysis filter pair. First low pass filter is applied for each row of data, to obtain low frequency components of the rows. It being a half band filter, the output of LPF contains only frequencies in the first half of the original frequency range. Similarly high pass filter is applied for the same 
rows of data and now the high pass components are separated and placed by the side of the low pass components.

The application of wavelet transform to an image, the obtained approximation sub signal shows the general trend of pixel values and three detail sub signals show the vertical, horizontal and diagonal details or changes in the image. This can be repeated till required level of decomposition is reached. The filters have the effect of separating out finer and finer details. This filtering process using wavelet can be understood from the Figure 1 to Figure 3.

\begin{tabular}{|l|l|}
\hline LL & HL \\
\hline LH & HH \\
\hline
\end{tabular}

Figure $1.1^{\text {st }}$ Level Decomposition

\begin{tabular}{|c|c|c|}
\hline LL & HL & \multirow{2}{*}{ HL } \\
\cline { 1 - 1 } LH & HH & \\
\hline \multicolumn{2}{|c|}{ LH } & HH \\
\hline
\end{tabular}

Figure 2. $2^{\text {nd }}$ Level decomposition

\begin{tabular}{|l|l|l|l|}
\hline & HL & \multirow{2}{*}{ HL } & \multirow{2}{*}{ HL } \\
\cline { 1 - 2 } LH & HH & \\
\cline { 1 - 1 } LH & HH & \\
\hline \multicolumn{2}{|c|}{ LH } & HH \\
\hline
\end{tabular}

Figure 3. $3^{\text {rd }}$ Level Decomposition

In $2 \mathrm{D}$, the images are considered to be matrices with $\mathrm{M}$ rows and $\mathrm{N}$ columns. At every level four sub images are obtained, the approximation, the vertical detail, the horizontal detail and the diagonal detail. To get the next level of decomposition the approximation detail is used. The ship ISAR image and its decomposition up to $3^{\text {rd }}$ level using wavelet transform are shown in Figure 4 and Figure 5.

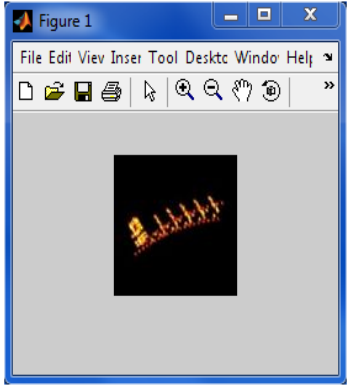

Figure 4. ISAR Image of a ship

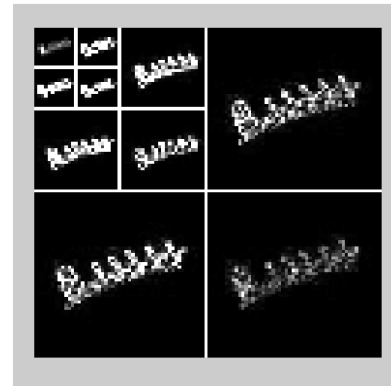

Figure 5. ISAR Image of a ship

The wavelet transform is applied to the set of ISAR images considered. The approximation, horizontal, vertical and diagonal details are computed. The wavelet transform is applied to the approximation detail up to $3^{\text {rd }}$ level to compute the details at each level. The approximation, horizontal, vertical and diagonal details and their combination at each level are taken independently one set at a time. Principal component analysis is applied to each set of values. In PCA, the above mentioned feature values of each set are arranged in the form of a matrix. Then covariance matrix is computed. The Eigen values of the covariance matrix are computed and used as a feature vector. The classification performance of all the combinations of wavelet coefficients taken as feature vectors is computed for the considered data set of measured ship ISAR images.

\subsection{Watershed Transform}

Direct application of Watershed transform to a gradient image causes over segmentation. The concept of markers is used to control over segmentation. A marker is a connected component belonging to an image. First a set of internal markers that are inside each of the object of interest and a set of external markers that are contained in the background are to BE identified. These markers are used to modify the gradient image. The toolbox function imextendedmin $(f, h)$ is used to compute the set of low spots in the image that are deeper than their surroundings by a certain height threshold $\mathrm{h}$ to obtain the internal marker image im. The next step is to find the external markers or pixels that belong to the back ground by applying Watershed transform of the distance transform of the internal marker image im. Thus obtained internal and external markers are used to modify the gradient image by imposing regional minima at the locations of internal and external markers using the following two MATLAB program statements. 
$\mathrm{g} 2=$ imimposemin $(\mathrm{g}, \mathrm{im} \mid \mathrm{em})$

L2=watershed $(\mathrm{g} 2)$

The g2 is the marker modified gradient image. Finally the Watershed transform of the marker modified gradient image is computed to obtain L2. With the below program statement, the Watershed ridge lines are superimposed on the original image to achieve much improved segmentation.

$\mathrm{F}(\mathrm{L} 2==0)=255$ Figure 7.

The ISAR image of a ship and the image with improved segmentation are shown in Figure 6 and

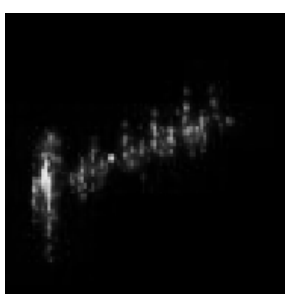

Figure 6. Ship ISAR Image in Binary Format

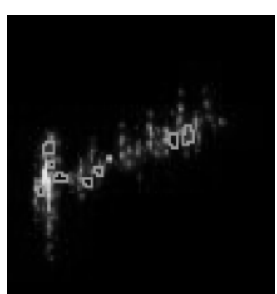

Figure 7. Segmented Ship ISAR Image in Binary Format

Now wavelet transform is applied to the image with improved segmentation achieved through Watershed s egmentation up to third level. The obtained detail matrix at each level is used as a feature vector and given as input to the classifier. The approximation, horizontal, vertical and diagonal detail matrices computed up to third level decomposition are taken and principal component analysis is applied and computed Eigen values are used as feature vectors to be given as input to the classifier. The combination of approximation and other three details (the complete set of wavelet coefficients) computed up to third level is also given as input to the classifier and the classification performance is computed.

\subsection{RGB Images}

The color images in RGB format are taken. An image in RGB format can be considered as a stack of three two dimensional matrices. The three images forming an RGB color image are the red, green and blue component images. If the RGB image belongs to a class double, the range of pixel intensity values is [0, 1]. If the RGB image is of class unit8, the range of pixel values lie between 0 and 255 . For unit 16 class image, the range of pixel values will be $[0,66555]$. The number of bits that represent the pixel values of the component image is called the bit depth of the RGB image. When the component image is a two bit image, the corresponding depth of the RGB image is 24 bits deep. If $\mathrm{b}$ is the number of bits in each component image then the number of colors in an image is $\left(2^{\mathrm{b}}\right)^{3}$.

The R, G, B component image matrices are extracted from the RGB image matrix. For the obtained each component image matrix, the mean and standard deviation are computed. The values obtained from the three component images are combined to form a feature vector that has given good classification results. Similarly, the component matrices are concatenated in combinations $R \& G, G \& B$ and $R \& B$. The mean, median and standard deviation values are computed for the combined component matrices (RG, GB, and $\mathrm{RB})$. The obtained statistical values of the component images R, G, B and combinations RG, GB and RB are given as a feature vectors to the neural network for classification. The two feature vectors discussed are given below:

$$
\begin{array}{ll}
M e a n_{-} R & S t d \_R \\
M e a n_{-} G & S t d \_G \\
M e a n_{-} B & S t d \_B \\
M e a n_{-} R G & S t d \_R G \\
M e a n_{-} G B & S t d \_G B \\
M e a n_{-} R B & S t d \_R B
\end{array}
$$

The columns of the matrix are concatenated to form a single column feature vector since the input to the neural network based classifier is required to be single dimensional vector. In both the cases the length of the feature vector is 6 . 


\section{DATA SET GENERATION}

In this work, the data set is generated by rotating the ship ISAR images obtained from literature. The performance of classification of each of the feature vectors discussed in previous sections is computed for two ships ISAR images measured using Instrumentation Radar. There are twelve images for each of the two ships. So there are twenty four images in the training set and test set independently. The classification performance for each of the feature vector using the artificial neural network is given in Table 1 to Table 9 .

\section{FEATURE VECTOR DESCRIPTION}

The size of the images in training and test set is 30x30. Except for RGB images, each image is resized to 3. In case of Wavelet coefficients, the size of the combined matrix of approximation, horizontal, vertical and diagonal coefficients is $15 \times 60,8 \times 32$ and $4 \times 16$ for the three levels of decomposition resp. So the size of the computed feature vector after application of PCA is 900, 256 and 128 for the first, second and third level decomposition. The size of the individual approximation, horizontal, vertical or diagonal details is $15 \times 15,8 \times 8$ and $4 \times 4$ for the three levels rasp... So the size of the final feature vector when approximation or horizontal or vertical or diagonal detail alone used is 256, 64 and 16 respectively for the one, two and three decompositions respectively. The size of the feature vectors is same when images with Watershed segmentation are used.

In case of RGB images, the size of the image is $100 \times 100 \times 3$. The size of the each component image is $100 \times 100$.The feature vectors obtained from individual $R, G, B$ images or combined images RG, GB, RB the feature vector given by (2) and (3). The mean and standard deviation values are computed for the ship ISAR images shown in Figure 8 to Figure 11 of two targets at two different aspect angles.

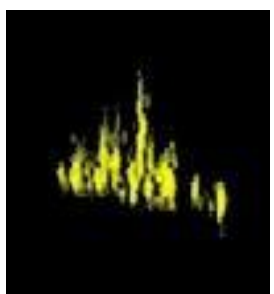

Figure 8. Target 1 ISAR image at aspect $0^{0}$

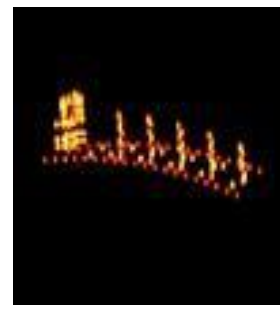

Figure 9. Taget 2 ISAR image at aspect $0^{0}$

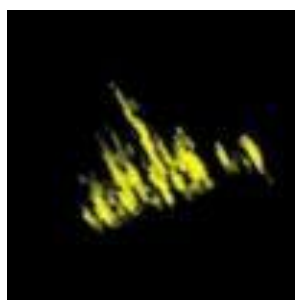

Figure 10. Target 1 ISAR image at aspect $30^{\circ}$

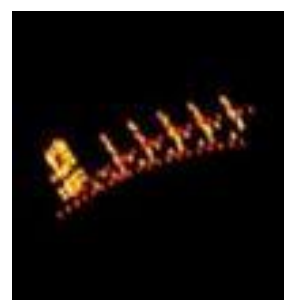

Figure 11. Target 2 ISAR image at aspect $30^{\circ}$

The computed statistical moment values for single R,G,B component images and combined $\mathrm{RG}, \mathrm{GB}, \mathrm{RB}$ of the ISAR images at aspect angle $0^{\circ}$ and $30^{\circ}$ of the two targets shown are given in Tables 1 and Table 2 .

Table 1. Statistical Moments of Single Component Images(R, G, B) for the Two Targets

\begin{tabular}{cccc}
\hline \multicolumn{2}{c}{ At aspect angle $0^{\circ}$} & \multicolumn{2}{c}{ At aspect angle $30^{\circ}$} \\
Target 1 & Target 2 & Target 1 & Target 2 \\
\hline 15.0447 & 14.1162 & 14.9728 & 14.1288 \\
30.0284 & 25.1107 & 27.6775 & 22.2324 \\
6.0493 & 5.0322 & 5.7158 & 4.7776 \\
12.5120 & 11.6661 & 10.6786 & 9.4176 \\
15.3883 & 7.7932 & 15.3415 & 7.6413 \\
30.9825 & 19.8523 & 28.3539 & 16.6296 \\
\hline
\end{tabular}

Table 2. Statistical Moments of Component Images Combined (RG, GB, and RB)

\begin{tabular}{cccc}
\hline \multicolumn{2}{c}{ At aspect angle $0^{\circ}$} & \multicolumn{2}{c}{ At aspect angle $30^{\circ}$} \\
Target 1 & Target 2 & Target 1 & Target 2 \\
\hline 10.5470 & 9.5742 & 10.3443 & 9.4529 \\
12.3862 & 9.5068 & 12.0200 & 9.0614 \\
10.7188 & 6.4127 & 10.5286 & 6.2095 \\
13.0606 & 5.7885 & 12.4984 & 5.0997 \\
15.2165 & 10.9547 & 15.1572 & 10.8848 \\
0.6747 & 3.7183 & 0.4783 & 3.9618 \\
\hline
\end{tabular}




\section{CLASSIFICATION PERFORMANCE}

Classification is done using Probabilistic neural network. MATLAB neural network toolbox is used. The classification performance is calculated from the number of successful classifications and the total number of images tested. For each of the feature vector studied in this paper, the classification is tested for the ISAR images of two ship targets measured using high resolution radar and performance is given in Table 3 to Table 8. The classification performance of any feature vector specified in this work is not same for the two targets considered.

The classification performance when wavelet coefficients, approximation and the other three details combined together used as feature vector for ISAR images with and without segmentation for three level decompositions is given in Table 3. Similarly the performance of the approximation, horizontal, vertical and diagonal details taken independently for the ship ISAR images with and without application of Watershed segmentation is given in Table 4 to Table 7.

The classification performance of color moments computed for single R, G, B component images and combined component images RG, GB and RB is given in Table 8.

Table 3. Classification Performance Percentage for the Approximation, Horizontal, Vertical and Diagonal Details Combined

\begin{tabular}{ccccccc}
\hline & \multicolumn{2}{c}{ Without Segmentation } & \multicolumn{3}{c}{ With segmentation } \\
Target & Level 1 & Level2 & Level3 & Level1 & Level2 & Level3 \\
\hline 1 & 58.3 & 25 & 25 & 33.3 & 50 & 83.3 \\
2 & 91.6 & 91.6 & 83.3 & 91.6 & 58.3 & 58.3 \\
\hline
\end{tabular}

Table 4. Classification Performance Percentage for the Approximation Details

\begin{tabular}{ccccccc}
\hline & \multicolumn{2}{c}{ Without Segmentation } & \multicolumn{3}{c}{ With Segmentation } \\
Target & Level 1 & Level 2 & Level 3 & Level 1 & Level 2 & Level 3 \\
\hline 1 & 33.3 & 41.6 & 33.3 & 50 & 50.6 & 58.3 \\
2 & 41.6 & 91.6 & 91.6 & 58.3 & 33.3 \\
\hline
\end{tabular}

Table 5. Classification Performance Percentage for the Horizontal Details

\begin{tabular}{ccccccc}
\hline & & Without Segmentation & \multicolumn{3}{c}{ With Segmentation } \\
Target & Level 1 & Level 2 & Level 3 & Level 1 & Level 2 & Level 3 \\
\hline 1 & 91.6 & 66.6 & 66.6 & 66.6 & 66.6 & 41.6 \\
2 & 75 & 66.6 & 75 & 33.3 & 33.3 & 66.6 \\
\hline
\end{tabular}

Table 6. Classification Performance Percentage for the Vertical Details

\begin{tabular}{ccccccc}
\hline & \multicolumn{3}{c}{ Without Segmentation } & \multicolumn{3}{c}{ With Segmentation } \\
Target & Level 1 & Level 2 & Level 3 & Level 1 & Level 2 & Level 3 \\
\hline 1 & 66.6 & 50 & 66.6 & 75 & 58.3 & 75 \\
2 & 33.3 & 58.3 & 58.3 & 41.6 & 58.3 & 41.6 \\
\hline
\end{tabular}

Table 7. Classification Performance Percentage for the Diagonal Details

\begin{tabular}{ccccccc}
\hline & \multicolumn{3}{c}{ Without Segmentation } & \multicolumn{3}{c}{ With Segmentation } \\
Target & Level 1 & Level 2 & Level 3 & Level 1 & Level 2 & Level 3 \\
\hline 1 & 58.3 & 58.3 & 83.3 & 33.3 & 75 & 50 \\
2 & 58.3 & 50 & 66.6 & 50 & 33.3 & 58.3 \\
\hline
\end{tabular}

Table 8. Classification Performance Percentage of the Color Moments

\begin{tabular}{ccc}
\hline Computed for Single R, G, B Components & Computed for Two Components RG, \\
GB, RB & 91.6 \\
\hline Target 1 & 66.6 & 75 \\
Target 2 & 86.33 & 75 \\
\hline
\end{tabular}

\section{CONCLUSION}

In this work, we compared the classification performance of the different feature vectors i.e. wavelet coefficients combined and individual at different levels of decomposition and statistical moments for the measured ship ISAR image data set. The performance is given considering the classification of both the 
targets considered. From the Table 3 to able 8 , it is observed that the whole set of wavelet coefficients (approximation, horizontal, vertical and diagonal) at first level decomposition has shown satisfactory classification performance for the ISAR images without segmentation (Table 3). This combined set of wavelet coefficients computed at third level decomposition has shown satisfactory classification performance for ISAR images with segmentation (Table 3). The approximation details are not good for classification of ISAR images with and without segmentation (Table 4). Whereas the horizontal detail coefficients computed at all the three level decompositions have shown good classification performance for ISAR images without segmentation (Table 5). The vertical detail coefficients computed at third level and second level decomposition have classified the ISAR images without segmentation and with segmentation respectively up to the level of acceptance (Table 6). The diagonal details computed at first and third level decomposition have classified the ISAR images without segmentation satisfactorily (Table 7). From this study it is understood that the wavelet coefficients have shown better classification performance for the ship ISAR image data set without segmentation than with segmentation. The statistical moments have shown good classification performance for the considered ISAR image data set (Table 8). Among the different wavelet coefficients considered, the horizontal coefficients computed at level 1 decomposition have shown the best classification performance. We can see that, out of all the feature vectors considered in this work, the statistical moments computed from the combined component images RG, GB and RB is the most suitable feature vector for ship ISAR image classification since the additional advantage with this feature vector is the small length of the feature vector irrespective of the image size. The feature vectors studied in this paper can be used for classification of images of different applications. The performance will vary when the data set changes.

\section{REFERENCES}

[1] I. Jordanov and A. G. Nuffield, "Feed Forward Neural Networks for Automated Classification," in Proc. 9th IEEE Int. Conf. on Cognitive Informatics (ICCI'10), 2010.

[2] R. S. ChorasImage, "Feature Extraction Techniques and Their Applications for CBIR and Biometrics Systems," International journal of biology and biomedical engineering, vol/issue: 1(1), 2007.

[3] A. Chadha, et al., "Comparative Study and Optimization of Feature- Extraction Techniques for Content based Image Retrieval," International Journal of Computer Applications, vol/issue: 52(20), pp. 0975-8887, 2012.

[4] D. P. Tian, et al., "A Review on Image Feature Extraction and Representation Techniques," International Journal of Multimedia and Ubiquitous Engineering, vol/issue: 8(4), 2013.

[5] S. Sivaperumal and M. Sundhararajan, "Advance feature extraction of MRI Brain Image and Detection using Local Segmentation Method with Watershed," International Journal of Electrical and Electronics Engineering Research (IJEEER), vol/issue: 3(4), pp. 87-94, 2013.

[6] P. Zhang, et al., "A New Framework of the Unsupervised Classification for High-Resolution Remote Sensing Image," TELKOMNIKA, vol/issue: 10 (7), pp. 1746 1755, 2012.

[7] K. Anuradha and K. Sankaranarayanan, "Comparison of Feature Extraction Techniques to classify Oral Cancers using Image Processing," International Journal of Application or Innovation in Engineering \& Management (IJAIEM), vol/issue: 2(6), 2013.

[8] Zaheeruddin, et al., "Detection and Shape Feature Extraction of Breast Tumor in Mammograms," in Proc. of WCE, London, U.K., 2012.

[9] M. B. Tayel and M. E. E. Bouridy, "ECG Images Classification using Artificial Neural Network Based on Several Feature Extraction Methods," IEEE, 2008.

[10] A. Phinyomark, et al., "Feature Extraction and Reduction of Wavelet Transform Coefficients for EMG Pattern Classification," Electronics and Electrical Engineering, vol/issue: 6(122), 2012.

[11] Kheder G., et al., "Feature extraction by wavelet transforms to analyze the heart rate variability during two meditation technique," in 6th WSEAS Int. Conf. on Circuits, Systems, Electronics, Control \& Signal Processing, Cairo, Egypt., pp. 374, 2007.

[12] M. Nazarloo, et al., "Gender Classification Using Hybrid of Gabor Filters and Binary Features of an Image," International Journal of Electrical and Computer Engineering (IJECE), vol/issue: 4(4), pp. 539 547, 2014.

[13] R. C. Gonzalez, et al., "Color Image Processing, Wavelets and Image Segmentation," in Digital Image Processing Using MATLAB, $2^{\text {nd }}$ ed., New Delhi, Tata McGraw-Hill, pp. 272-330, 331-373,489-550, 2010.

[14] R. V. R. Chary, et al., "Feature Extraction Methods for Color Image Similarity," Advanced Computing: International Journal, vol/issue: 3(2), 2012.

[15] S. Mahajan and D. Patil, "Comparison of Color and Color with Edge Feature Extraction Using Contribution-based Clustering Algorithm," in 4th Int. Conf. on Communication Systems and Network Technologies, 2014. 